(2) Open Access Full Text Article

ORIGINALRESEARCH

\title{
Safety and Efficacy of Alpha Lipoic Acid During 4 Years of Observation: A Retrospective, Clinical Trial in Healthy Subjects in Primary Prevention
}

This article was published in the following Dove Press journal:

Drug Design, Development and Therapy

\author{
Giuseppe Derosa $\mathbb{1}^{1,2}$ \\ Angela D'Angelo $\mathbb{D}^{2}$ \\ Paola Preti ${ }^{1}$ \\ Pamela Maffioli ${ }^{1}$ \\ 'Department of Internal Medicine and \\ Therapeutics, University of Pavia, Pavia, \\ Italy; ${ }^{2}$ Laboratory of Molecular Medicine, \\ University of Pavia, Pavia, Italy
}

\begin{abstract}
Aim: To evaluate the safety of four different dosages of alpha lipoic acid (400, 600, 800, and $1200 \mathrm{mg}$ ) as food supplement on adverse events related to alpha lipoic acid consumption and efficacy on glycemic status and lipid profile in subjects with euglycemia or dysglycemia.

Methods: We conducted a retrospective, observational study enrolling 322 patients, 83 taking $400 \mathrm{mg} /$ day, 78 taking $600 \mathrm{mg} /$ day, 80 taking $800 \mathrm{mg} /$ day, and 81 taking $1200 \mathrm{mg} /$ day alpha lipoic acid, respectively.

Results: In the groups treated with alpha lipoic acid 800 and $1200 \mathrm{mg} / \mathrm{day}$, we registered a reduction of FPG, TC, LDL-C, and Tg compared to baseline ( $p<0.05$ for all with alpha lipoic acid $800 \mathrm{mg} /$ day, and $\mathrm{p}<0.01$ for all with alpha lipoic acid $1200 \mathrm{mg} /$ day). The values recorded in the group treated with alpha lipoic acid $1200 \mathrm{mg}$ /day were significantly lower compared to the ones obtained with alpha lipoic acid $400 \mathrm{mg}$ /day. Moreover, alpha lipoic acid $1200 \mathrm{mg} /$ day reduced HsCRP levels compared to baseline and compared to $400 \mathrm{mg}$ /day ( $<0.05$ for both). In the group treated with alpha lipoic acid at $800 \mathrm{mg}$ /day, 5 subjects with IFG and 1 subject with IGT returned euglycemic. In the group treated with alpha lipoic acid at $1200 \mathrm{mg} /$ day, 11 subjects with IFG and 3 subjects with IGT returned euglycemic. Adverse events of patients during alpha lipoic acid treatment included nausea, vomiting, dizziness, cutaneous rash, hypoglycemia, and hypotension. Adverse events did not differ among the four groups.
\end{abstract}

Conclusion: The chronic use (4 years) of a food supplement containing alpha lipoic acid is well tolerated, without significant differences between lower and higher dosages and improves glycemic status and lipid profile but only if administered at high dosage.

Keywords: alpha lipoic acid, dysglycemia, efficacy, safety

\section{Introduction}

Type 2 diabetes mellitus is a risk factor for cardiovascular disease, ${ }^{1}$ but dysglycemia could be a cardiovascular risk also in nondiabetic patients. A meta-analysis of 20 studies conducted by Coutinho et $\mathrm{al}^{2}$ including 95,783 nondiabetic subjects, and during a mean follow-up of 12.4 years, reported 3707 cardiovascular events in these subjects. These Authors suggested a relationship between the risk of cardiovascular events and both fasting and post-prandial glucose levels. Such a relationship extended below diagnostic blood glucose levels for impaired fasting glucose (IFG) or impaired glucose tolerance (IGT).

Pre-diabetic dysglycemia acts together with other cardiovascular risk factors; usually dysglycemic patients are also affected by insulin resistance and hyperinsulinemia, elevated blood pressure, high triglycerides, low high-density lipoprotein-cholesterol
Department of Internal Medicine and

Therapeutics, University of Pavia, via Aselli,

43/45, Pavia 27100, Italy

Tel +39-0382 526217

Fax +39-0382 526259

Email giuseppe.derosa@unipv.it 
(HDL-C), small and dense low-density lipoprotein, central adiposity, elevated plasminogen activator inhibitor-1, and lowgrade inflammation. ${ }^{3}$ Experimental evidence supports a main role of impaired glucose homeostasis on the atherogenic process. Post-prandial hyperglycemia is usually the earliest sign of impaired glucose homeostasis. Higher and persistent elevation of post-prandial plasma glucose levels contributes significantly to overall glycemic exposure of body tissues, favoring atherosclerosis development.

Alpha lipoic acid also known as 1,2-dithiolane-3-pentanoic acid or thioctic acid is a naturally occurring compound synthesized enzymatically in plant and animal mitochondria from octanoic acid and cysteine. ${ }^{4}$ Alpha lipoic acid is mainly known for its anti-oxidant activity; ${ }^{4,5}$ furthermore, it also has several other properties: actions include anti-inflammatory activity, chelation of metal ions and effects on glucose and lipid metabolism. ${ }^{4}$ Alpha-lipoic acid has been reported to increase glucose disposal in diabetic states, in particular, lipoic acid increases glucose uptake by skeletal muscles of obesediabetic ob/ob mice, ${ }^{6}$ however, long-term studies aimed to confirm the effects of alpha lipoic acid on glycemia and lipid profile in humans are lacking, as well as whether there are side effects and unwanted effects in patients taking alpha lipoic acid.

For this reason, the aim of this study was to evaluate the efficacy of four different dosages of alpha lipoic acid $(400,600,800$, and $1200 \mathrm{mg})$ on glycemic status and lipid profile in subjects with euglycemia (defined by glycemia $<$ $100 \mathrm{mg} / \mathrm{dl}$ ) or dysglycemia (defined by glycemia between 100 and $125 \mathrm{mg} / \mathrm{dl}$ ). Secondary objective was monitoring adverse events related to alpha lipoic acid consumption.

\section{Materials and Methods}

\section{Study Design}

This retrospective, observational study was conducted at the Department of Internal Medicine and Therapeutics of the University of Pavia in Pavia, Italy.

The study protocol was approved by the review board of Fondazione IRCCS Policlinico San Matteo, PAVIA, Italy (P-2017000837) and was conducted in accordance with the Declaration of Helsinki and its amendments. Suitable patients, identified from review of case notes and/or computerized clinic registers were contacted personally or by telephone. All eligible candidates had to provide signed informed consent before enrolling in the study.

\section{Patients}

Study was based on the selection of data from a considerable amount of clinical records, regarding Caucasian patients, aged $\geq 18$ years of either sex with euglycemia or a condition of IFG or IGT treated with alpha lipoic acid over the last four years. Patients' information was obtained by means of the medical record review.

Subjects with high glucose levels variability in the year preceding the study ( $\pm 20 \%$ ), diabetic patients, patients with abnormal thyroid function, patients with hepatic impairment (defined as transaminases greater than three times the maximum limits laboratory) or renal impairment (defined as creatinine values greater than the upper limit of the laboratory) were excluded. Patients taking drugs potentially affecting glucose metabolism were also excluded. Also, subjects with diseases such as cancer, chronic inflammatory diseases (rheumatic and infectious), psychiatric diseases were excluded. Patients with serious cardiovascular disease (CVD) (eg, New York Heart Association class I-IV congestive heart failure or a history of myocardial infarction or stroke) or cerebrovascular conditions within 6 months before study enrollment also were excluded. Women who were pregnant or breastfeeding or of childbearing potential and not taking adequate contraceptive precautions were also excluded.

\section{Assessments}

We collected information about medical history, body weight, height, body mass index (BMI), systolic (SBP) and diastolic blood pressure (DBP) fasting plasma glucose (FPG), total cholesterol (TC), HDL-C, triglycerides (Tg), low-density lipoprotein-cholesterol (LDL-C), high sensitive C-reactive protein (Hs-CRP). These parameters were assessed at baseline, and after 4 years. All parameters were determined in fasting state, after a 12 -h overnight fast, in the plasma. All measurements were performed in a central laboratory.

BMI was calculated by the investigators as weight in kilograms divided by the square of height in meters.

Blood pressure (BP) measurements were obtained from each patient (using the right arm) in the seated position, using a standard mercury sphygmomanometer (Erkameter 3000, ERKA, Bad Tolz, Germany) (Korotkoff I and V) with a cuff of appropriate size. BP was measured by the same investigator at each visit, in the morning, after the patient had rested for $>10$ minutes in a quiet room. Three successive BP readings were obtained at 1-minute intervals, and the mean of the 3 readings was calculated. 
Plasma glucose was assayed by glucose-oxidase method (GOD/PAP, Roche Diagnostics, Mannheim, Germany) with intra- and interassay coefficients of variation $(\mathrm{CsV})$ of $<2 \%{ }^{7}$

Total cholesterol and Tg levels were determined using fully enzymatic techniques ${ }^{8,9}$ on a clinical chemistry analyzer (HITACHI 737; Hitachi, Tokyo, Japan); intra- and interassay $\mathrm{CsV}$ were 1.0 and 2.1 for TC measurement, and 0.9 and 2.4 for $\mathrm{Tg}$ measurement, respectively. Highdensity lipoprotein-cholesterol level was measured after precipitation of plasma apo B-containing lipoproteins with phosphotungstic acid ${ }^{10}$ intra- and interassay $\mathrm{CsV}$ were 1.0 and 1.9, respectively; LDL-C level was calculated by the Friedewald formula. ${ }^{11}$

High-sensitivity C-reactive protein was measured with the use of latex-enhanced immunonephelometric assays on a BN II analyser (Dade Behring, Newark, Delaware,
USA). The intra- and interassay $\mathrm{CsV}$ were $5.7 \%$ and $1.3 \%$, respectively. ${ }^{12}$

\section{Alpha Lipoic Acid Treatment}

The various dosages of alpha lipoic acid taken into account were: $400 \mathrm{mg}$ (fast slow release), $600 \mathrm{mg}$ (normal release), $800 \mathrm{mg}$ (fast slow release), and $1200 \mathrm{mg}$ (normal release).

\section{Statistical Analysis}

Quantitative variables were described as mean and standard deviation if normally distributed and with median multiple interquartile ranges if they have an asymmetric distribution. Qualitative variables were presented with counts and percentages.

Comparison among different alpha lipoic acid dosage, was performed using $\mathrm{x} 2$-test for categorical variables. All

Table I Baseline Patient Characteristics

\begin{tabular}{|c|c|c|c|c|}
\hline Parameters & \multicolumn{4}{|c|}{ Alpha Lipoic Acid } \\
\hline \multicolumn{5}{|l|}{$\mathrm{N}$ of pts (322) } \\
\hline Dose (mg) & 400 & 600 & 800 & 1200 \\
\hline $\mathrm{N}$ & 83 & 78 & 80 & 81 \\
\hline Age (years) & $51.6 \pm 9.2$ & $50.3 \pm 8.7$ & $54.8 \pm 10.6$ & $55.2 \pm 10.9$ \\
\hline$M / F$ & $40 / 43$ & $38 / 40$ & $39 / 41$ & $4 I / 40$ \\
\hline Smoking status (M/F) & $11 / 13$ & $14 / 13$ & $|2 /| 1$ & $9 / 11$ \\
\hline Weight (kg) & $75.2 \pm 6.6$ & $74.9 \pm 6.4$ & $75.1 \pm 6.7$ & $75.4 \pm 7.0$ \\
\hline Height (m) & $1.67 \pm 0.09$ & $1.67 \pm 0.09$ & $1.66 \pm 0.08$ & $1.65 \pm 0.07$ \\
\hline BMI $\left(\mathrm{kg} / \mathrm{m}^{2}\right)$ & $27.1 \pm 2.1$ & $26.8 \pm 1.9$ & $27.2 \pm 2.2$ & $27.7 \pm 2.5$ \\
\hline $\mathrm{SBP}(\mathrm{mmHg})$ & $136.2 \pm 6.1$ & $135.8 \pm 5.9$ & $136.9 \pm 6.7$ & $135.7 \pm 5.7$ \\
\hline $\mathrm{DBP}(\mathrm{mmHg})$ & $82.1 \pm 4.6$ & $81.8 \pm 4.2$ & $82.3 \pm 4.7$ & $82.5 \pm 4.9$ \\
\hline FPG (mg/dl) & $95.1 \pm 12.1$ & $95.4 \pm 12.4$ & $94.6 \pm 11.8$ & $94.8 \pm 11.9$ \\
\hline $\mathrm{TC}(\mathrm{mg} / \mathrm{dl})$ & $216.1 \pm 14.8$ & $215.6 \pm 14.5$ & $214.4 \pm 13.7$ & $215.9 \pm 14.8$ \\
\hline LDL-C (mg/dl) & $147.3 \pm 8.5$ & $146.3 \pm 8.2$ & $144.6 \pm 7.5$ & $146.8 \pm 8.6$ \\
\hline HDL-C (mg/dl) & $42.5 \pm 5.8$ & $42.7 \pm 5.9$ & $43.1 \pm 6.1$ & $42.4 \pm 5.7$ \\
\hline $\mathrm{Tg}(\mathrm{mg} / \mathrm{dl})$ & $131.6 \pm 15.8$ & $133.1 \pm 16.3$ & $133.6 \pm 16.8$ & $133.5 \pm 16.7$ \\
\hline $\mathrm{Hs}-\mathrm{CRP}(\mathrm{mg} / \mathrm{l})$ & $1.3 \pm 0.5$ & $1.4 \pm 0.6$ & $1.3 \pm 0.5$ & $1.5 \pm 0.7$ \\
\hline
\end{tabular}

Note: Data are expressed as mean \pm standard deviations.

Abbreviations: M, males; F, females; BMI, body mass index; SBP, systolic blood pressure; DBP, diastolic blood pressure; FPG, fasting plasma glucose; TC, total cholesterol; LDL-C, low-density lipoprotein-cholesterol; HDL-C, high-density lipoprotein-cholesterol; Tg, triglycerides; Hs-CRP, high-sensitivity C-reactive protein. 
Table 2 Medications Taken at Baseline in the Alpha Lipoic Acid Groups

\begin{tabular}{|c|c|c|c|c|}
\hline $\begin{array}{l}\text { Dose, mg } \\
\text { Patients, n }\end{array}$ & $\begin{array}{l}400 \\
83\end{array}$ & $\begin{array}{l}600 \\
78\end{array}$ & $\begin{array}{l}800 \\
80\end{array}$ & $\begin{array}{l}1200 \\
81\end{array}$ \\
\hline \multicolumn{5}{|c|}{ Antihypertensives, n (\%) } \\
\hline Diuretic & $8(10.1)$ & $6(8.3)$ & $5(7.0)$ & $9(12.0)$ \\
\hline ACE-I & II (13.9) & $10(13.9)$ & $9(12.7)$ & $12(16.0)$ \\
\hline Sartan & $9(I I .4)$ & $13(18.1)$ & $8(11.3)$ & $10(13.3)$ \\
\hline Ca-antagonist & $7(8.9)$ & $6(8.3)$ & $8(11.3)$ & $7(9.3)$ \\
\hline$\beta$-blocker & $3(3.8)$ & $2(2.8)$ & $2(2.8)$ & $3(4.0)$ \\
\hline \multicolumn{5}{|c|}{ Hypocholesterolemics, n (\%) } \\
\hline Statin & $16(20.3)$ & $15(20.8)$ & $13(18.3)$ & $18(24.0)$ \\
\hline Fibrate & $5(6.3)$ & $4(5.6)$ & $4(5.6)$ & $6(8.0)$ \\
\hline Ezetimibe & $6(7.6)$ & $7(9.7)$ & $9(12.7)$ & $7(9.3)$ \\
\hline Omega-3 & $4(5.1)$ & $5(5.6)$ & $4(5.6)$ & $2(2.7)$ \\
\hline PCSK9-I & $3(3.8)$ & $2(2.8)$ & I (I.4) & $\mathrm{I}(\mathrm{I} .3)$ \\
\hline \multicolumn{5}{|c|}{ Antiarrhythmics, n (\%) } \\
\hline Amiodarone & $2(2.5)$ & I (I.4) & 0 & 0 \\
\hline Digoxin & $\mathrm{I}(\mathrm{I} .3)$ & 0 & I (I.4) & 0 \\
\hline \multicolumn{5}{|l|}{ PPI, n (\%) } \\
\hline Omeprazole & $3(3.8)$ & $4(5.6)$ & $2(2.8)$ & $\mathrm{I}(\mathrm{I} .3)$ \\
\hline Pantoprazole & $2(2.5)$ & I (I.4) & 0 & 0 \\
\hline \multicolumn{5}{|c|}{ Antiaggregants, n (\%) } \\
\hline ASA & $3(3.8)$ & $4(5.6)$ & $2(2.8)$ & I (I.3) \\
\hline Clopidogrel & $2(2.5)$ & I (I.4) & 0 & 0 \\
\hline
\end{tabular}

Notes: $n$, number of patients; \%, percentage of patients.

Abbreviations: ACE-I, angiotensin-converting enzyme-inhibitors; PCSK9-I, proprotein convertase subtilisin/kexin type 9-inhibitors; PPI, proton pump inhibitors; ASA, acetylsalicylic acid.

statistical tests were carried out at a two-sided 5\% significance level.

Statistica 6.0 (Statsoft, Tulsa, OK, USA) was used for statistical computations. A p value of less than 0.05 was considered statistically significant. ${ }^{13}$

\section{Results}

\section{Study Sample}

A total of 322 patients were enrolled in this study, 83 taking $400 \mathrm{mg} /$ day, 78 taking $600 \mathrm{mg} /$ day, 80 taking $800 \mathrm{mg} /$ day, and 81 taking $1200 \mathrm{mg}$ /day alpha lipoic acid, respectively. Study population characteristic at baseline is described in Table 1. Medications taken at baseline, and after 4 years of alpha lipoic acid was described in Tables 2, and 3 .
Table 3 Medications Taken After 4 Years of Observation in the Alpha Lipoic Acid Groups

\begin{tabular}{|c|c|c|c|c|}
\hline $\begin{array}{l}\text { Dose, mg } \\
\text { Patients, n }\end{array}$ & $\begin{array}{l}400 \\
83\end{array}$ & $\begin{array}{l}600 \\
78\end{array}$ & $\begin{array}{l}800 \\
80\end{array}$ & $\begin{array}{l}1200 \\
81\end{array}$ \\
\hline \multicolumn{5}{|c|}{ Antihypertensives, $\mathbf{n}(\%)$} \\
\hline Diuretic & $7(8.9)$ & $6(8.3)$ & $4(5.6)$ & $10(13.3)$ \\
\hline ACE-I & $13(16.5)$ & $12(16.7)$ & $10(14.1)$ & $13(17.3)$ \\
\hline Sartan & $12(15.2)$ & $16(22.2)$ & $12(16.9)$ & $14(18.7)$ \\
\hline Ca-antagonist & $8(10.1)$ & $7(9.7)$ & $9(12.7)$ & $8(10.7)$ \\
\hline$\beta$-blocker & $2(2.5)$ & $3(4.2)$ & $2(2.8)$ & $2(2.7)$ \\
\hline \multicolumn{5}{|c|}{ Hypocholesterolemics, n (\%) } \\
\hline Statin & $18(22.8)$ & $16(22.2)$ & I5 (2I.I) & $18(24.0)$ \\
\hline Fibrate & $4(5.1)$ & $5(5.6)$ & $4(5.6)$ & $5(6.7)$ \\
\hline Ezetimibe & $9(11.4)$ & $10(13.9)$ & II (I5.5) & $10(13.3)$ \\
\hline Omega-3 & $5(6.3)$ & $4(5.6)$ & $6(8.5)$ & $4(5.3)$ \\
\hline PCSK9-I & $3(3.8)$ & $2(2.8)$ & I (I.4) & $\mathrm{I}(\mathrm{I} .3)$ \\
\hline \multicolumn{5}{|c|}{ Antiarrhythmics, n (\%) } \\
\hline Amiodarone & $2(2.5)$ & $2(2.8)$ & $2(2.8)$ & $\mathrm{I}(\mathrm{I} .3)$ \\
\hline Digoxin & $\mathrm{I}(\mathrm{I} .3)$ & 0 & 0 & 0 \\
\hline \multicolumn{5}{|l|}{ PPI, n (\%) } \\
\hline Omeprazole & $5(6.3)$ & $6(8.3)$ & $3(4.2)$ & $2(2.7)$ \\
\hline Pantoprazole & $2(2.5)$ & $2(2.8)$ & I (I.4) & $\mathrm{I}(\mathrm{I} .3)$ \\
\hline \multicolumn{5}{|c|}{ Antiaggregants, n (\%) } \\
\hline ASA & $5(6.3)$ & $6(8.3)$ & $3(4.2)$ & $2(2.7)$ \\
\hline Clopidogrel & $2(2.5)$ & $2(2.8)$ & I (I.4) & I (I.3) \\
\hline
\end{tabular}

Notes: $\mathrm{n}$, number of patients; \%, percentage of patients.

Abbreviations: ACE-I, angiotensin-converting enzyme-inhibitors; PCSK9-I, proprotein convertase subtilisin/kexin type 9-inhibitors; PPI, proton pump inhibitors; ASA, acetylsalicylic acid.

\section{Variation of Various Parameters During the 4 Years of Follow-Up}

During the 4 years of observation, we did not record any variation of metabolic parameters in the groups treated with 400 and $600 \mathrm{mg} /$ day of alpha lipoic acid, respectively. In the groups treated with alpha lipoic acid 800 , and $1200 \mathrm{mg} /$ day, instead, we registered a reduction of FPG, TC, LDL-C, and Tg compared to baseline ( $p<0.05$ for all with alpha lipoic acid $800 \mathrm{mg} /$ day, and $\mathrm{p}<0.01$ for all with alpha lipoic acid $1200 \mathrm{mg} /$ day) (Table 4). The values recorded in the group treated with alpha lipoic acid $1200 \mathrm{mg} /$ day were significantly lower compared to the ones obtained with alpha lipoic acid $400 \mathrm{mg} /$ day (Table 4). 
Table 4 Patient Characteristics After 4 Years of Follow-Up

\begin{tabular}{|c|c|c|c|c|}
\hline Parameters & \multicolumn{4}{|c|}{ Alpha Lipoic Acid } \\
\hline \multicolumn{5}{|l|}{$\mathrm{N}$ of pts (322) } \\
\hline Dose (mg) & 400 & 600 & 800 & 1200 \\
\hline $\mathrm{N}$ & 83 & 78 & 80 & 81 \\
\hline$M / F$ & $40 / 43$ & $38 / 40$ & $39 / 41$ & $41 / 40$ \\
\hline Smoking status (M/F) & $11 / 13$ & $14 / 13$ & $12 / 1 \mid$ & $9 / 11$ \\
\hline Weight (kg) & $74.8 \pm 6.2$ & $75.4 \pm 6.6$ & $76.3 \pm 7.1$ & $76.5 \pm 7.2$ \\
\hline Height (m) & $1.67 \pm 0.09$ & $1.67 \pm 0.09$ & $1.66 \pm 0.08$ & $1.65 \pm 0.07$ \\
\hline BMI $\left(\mathrm{kg} / \mathrm{m}^{2}\right)$ & $26.8 \pm 2.0$ & $27.0 \pm 1.9$ & $27.6 \pm 2.3$ & $28.1 \pm 2.6$ \\
\hline $\mathrm{SBP}(\mathrm{mmHg})$ & $133.1 \pm 5.9$ & $133.5 \pm 6.2$ & $134.1 \pm 6.4$ & $134.3 \pm 6.6$ \\
\hline $\mathrm{DBP}(\mathrm{mmHg})$ & $81.7 \pm 4.2$ & $81.4 \pm 4.0$ & $81.9 \pm 4.8$ & $82.1 \pm 5.0$ \\
\hline FPG (mg/dl) & $92.7 \pm 10.8$ & $90.1 \pm 10.2$ & $88.5 \pm 9.6^{*}$ & $83.1 \pm 8.8^{\circ \wedge}$ \\
\hline TC (mg/dl) & $207.3 \pm 12.4$ & $205.1 \pm 12.1$ & $197.5 \pm 11.8^{*}$ & $185.3 \pm 10.1^{\circ \wedge}$ \\
\hline LDL-C (mg/dl) & $139.9 \pm 8.9$ & $138.5 \pm 9.3$ & $132.4 \pm 7.9^{*}$ & $121.5 \pm 6.9^{\circ \wedge}$ \\
\hline HDL-C (mg/dl) & $42.6 \pm 5.9$ & $42.2 \pm 5.7$ & $42.8 \pm 6.0$ & $42.5 \pm 5.8$ \\
\hline $\mathrm{Tg}(\mathrm{mg} / \mathrm{dl})$ & $123.7 \pm 14.3$ & $121.9 \pm 13.8$ & $111.6 \pm 12.5^{*}$ & $106.4 \pm 10.6^{\circ \wedge}$ \\
\hline $\mathrm{Hs}-\mathrm{CRP}(\mathrm{mg} / \mathrm{l})$ & $1.2 \pm 0.4$ & $1.2 \pm 0.5$ & $I . I \pm 0.4$ & $0.9 \pm 0.3^{* \wedge}$ \\
\hline
\end{tabular}

Notes: Data are expressed as mean \pm standard deviations. ${ }^{*} \mathrm{p}<0.05$ vs baseline; ${ }^{\circ}<0.01$ vs baseline; ${ }^{\wedge} \mathrm{p}<0.05$ vs $400 \mathrm{mg}$

Abbreviations: M, males; F, females; BMI, body mass index; SBP, systolic blood pressure; DBP, diastolic blood pressure; FPG, fasting plasma glucose; TC, total cholesterol; LDL-C, low-density lipoprotein-cholesterol; HDL-C, high-density lipoprotein-cholesterol; Tg, triglycerides; Hs-CRP, high-sensitivity C-reactive protein.

Moreover, alpha lipoic acid $1200 \mathrm{mg} /$ day reduced HsCRP levels compared to baseline, and compared to $400 \mathrm{mg} /$ day $(\mathrm{p}<0.05$ for both) (Table 4).

\section{Glycemic Status Variation During the 4 Years of Observation}

In the group treated with alpha lipoic acid at $800 \mathrm{mg} / \mathrm{day}, 5$ subjects with IFG, and 1 subject with IGT returned euglycemic (Table 5). In the group treated with alpha lipoic acid at $1200 \mathrm{mg}$ /day, 11 subjects with IFG, and 3 subjects with IGT returned euglycemic (Table 5).

\section{Adverse Events During the 4 Years of Observation}

Adverse events of patients during alpha lipoic acid treatment included nausea, vomiting, dizziness, cutaneous rash, hypoglycemia, and hypotension. Adverse events did not differ among the four groups (Table 6).

\section{Discussion}

In our study, we did not observe significant side effects during the various dosages of alpha lipoic acid and we recorded an improvement of metabolic parameters with alpha lipoic acid at the dosage of 800 and $1200 \mathrm{mg} /$ day, suggesting that higher doses are needed to obtain an action on glycemic and lipid parameters. There are currently no comparative data in alpha lipoic acid assays in the literature. Our results are in line with a previous study conducted by our group, where we evaluated the effects of a nutraceutical containing, among other components, also alpha lipoic acid $600 \mathrm{mg}$ /day. ${ }^{5}$ Moreover, Akbari et al conducted a metaanalysis confirming our results: they also concluded 
Table 5 Glycemic Status Patients During Alpha Lipoic Acid Treatment

\begin{tabular}{|c|c|c|c|c|}
\hline \multirow{2}{*}{$\begin{array}{l}\text { Parameters } \\
\text { Dose (mg) }\end{array}$} & \multicolumn{4}{|c|}{ Alpha Lipoic Acid } \\
\hline & 400 & 600 & 800 & 1200 \\
\hline Patients & 83 & 78 & 80 & 81 \\
\hline$M / F$ & $40 / 43$ & $38 / 40$ & $39 / 41$ & $4 I / 40$ \\
\hline Smoking status (M/F) & $11 / 13$ & $14 / 13$ & $|2 / 1|$ & $9 / 11$ \\
\hline EU (n; \%) & $20 / 22$ & $18 / 20$ & $16 / 15$ & $15 / 14$ \\
\hline IFG (n; \%) & $8 / 7$ & $8 / 8$ & $11 / 10$ & $9 / 10$ \\
\hline IGT (n; \%) & $14 / 12$ & $11 / 13$ & $16 / 12$ & $18 / 15$ \\
\hline EU from IFG (n; \%) & $0 / 0$ & $\mathrm{I} / 0(\mathrm{I} .3)$ & $2 / 3(6.3)$ & $5 / 6(13.6)$ \\
\hline EU from IGT (n; \%) & $0 / 0$ & $0 / 0$ & $0 / 1(1.3)$ & $2 / 1(3.7)$ \\
\hline IGT from IFG (n: \%) & $0 / 0$ & $0 / 0$ & $0 / 0$ & $0 / 0$ \\
\hline D from IFG (n; \%) & $0 / 0$ & $0 / 0$ & $0 / 0$ & $0 / 0$ \\
\hline D from IGT (n; \%) & $0 / 0$ & $0 / 0$ & $0 / 0$ & $0 / 0$ \\
\hline Lost to FU from IFG (n; \%) & $0 / 0$ & $0 / 0$ & $0 / 0$ & $0 / 0$ \\
\hline Lost to FU from IGT (n; \%) & $0 / 0$ & $0 / 0$ & $0 / 0$ & $0 / 0$ \\
\hline
\end{tabular}

Abbreviations: M, males; F, females; IFG, impaired fasting glycemia; IGT, impaired glucose tolerance; EU, euglycemia; D, diabetes; FU, follow-up.

that alpha lipoic acid administration may lead to an improvement in glucose homeostasis parameters and lipid profiles with the exception of HDL-C levels. ${ }^{14}$

Regarding the effective dose necessary to obtain an improvement of glyco-metabolic parameters, in literature data are contrasting. In our current study, we recorded significant results only with alpha lipoic acid taken at higher dosage, 800 and $1200 \mathrm{mg} /$ day. This is in contrast with a previous study by our group, ${ }^{5}$ where we obtained positive results on glycemia and lipid profile, also with alpha lipoic acid at $600 \mathrm{mg} /$ day. Also, Okanović et al reported the efficacy of alpha lipoic acid at $600 \mathrm{mg} /$ day dosage in decreasing $\mathrm{Tg}$, but not glycemia or TC. ${ }^{15}$ These differences are probably due to the fact that in our current study we excluded type 2 diabetic patients, and included only patients with euglycemia, IFG, or IGT, differently from our previous study, ${ }^{5}$ and differently from Okanović et $\mathrm{al}_{1}{ }^{15}$ where only type 2 diabetic patients were included. As have been reported many times in literature, the higher is the baseline glycemia, the greatest will be the reduction expected with any treatment.
Given that type 2 diabetic patients have higher glycemia compared to euglycemic or dysglycemic ones, this is probably the reason that in type 2 diabetic patients alpha lipoic at the dosage of $600 \mathrm{mg}$ is enough to obtain a positive effect on metabolic parameters, while higher dosages are requested to improve glycemia in subjects with euglycemia or IFG or IGT condition.

Finally, considering alpha lipoic acid safety, no serious adverse events were recorded, and no differences among various dosages were observed regarding nausea, vomiting, dizziness, cutaneous rash, hypoglycemia, and hypotension. Our findings are in line with other previously published papers, for example Parente et $\mathrm{al}^{16}$ tested the safety of alpha lipoic acid at the dosage of $600 \mathrm{mg} / \mathrm{day}$ in pregnant women and no adverse events were reported in neither mothers or newborns.

The main limitation of this study is the observational design; however, the high numbers of patient involved and the long follow-up period could be the main strengths of this study. 
Table 6 Adverse Events of Patients During Alpha Lipoic Acid Treatment During the 4 Years of Observation

\begin{tabular}{|c|c|c|c|c|}
\hline \multirow[b]{2}{*}{ Dose (mg) } & \multicolumn{4}{|c|}{ Alpha Lipoic Acid } \\
\hline & 400 & 600 & 800 & 1200 \\
\hline Patients (322) (n) & 83 & 78 & 80 & 81 \\
\hline$M / F(I 58 / 164)(n)$ & $40 / 43$ & $38 / 40$ & $39 / 41$ & $4 I / 40$ \\
\hline \multicolumn{5}{|l|}{ EU patients (120) } \\
\hline Nausea (n; \%) & I/I (2.4) & I/I (2.6) & $2 / 0(2.5)$ & $\mathrm{I} / \mathrm{I}(2.5)$ \\
\hline Vomiting (n; \%) & I/I (2.4) & $0 / 1(1.3)$ & $\mathrm{I} / \mathrm{I}(2.5)$ & $\mathrm{I} / \mathrm{I}(2.5)$ \\
\hline Dizziness (n; \%) & $0 / 1(1.2)$ & I/I (2.6) & $\mathrm{I} / \mathrm{I}(2.5)$ & $0 / 1(1.2)$ \\
\hline Cutaneous rash (n; \%) & $0 / 0$ & I/0 (I.3) & $\mathrm{I} / \mathrm{I}(2.5)$ & $\mathrm{I} / \mathrm{I}(2.5)$ \\
\hline Hypoglycemia (n; \%) & $0 / 0$ & $0 / 0$ & $0 / 1(1.3)$ & $\mathrm{I} / \mathrm{I}(2.5)$ \\
\hline Hypotension (n; \%) & $0 / 0$ & $0 / 1(1.3)$ & I/0 (I.3) & $0 / 1(1.2)$ \\
\hline \multicolumn{5}{|l|}{ IFG patients (7I) } \\
\hline Nausea (n; \%) & $0 / 1(1.2)$ & $0 / 0$ & $\mathrm{I} / \mathrm{I}(2.5)$ & $0 / 1(1.2)$ \\
\hline Vomiting (n; \%) & $0 / 0$ & $0 / 0$ & $0 / 0$ & $0 / 0$ \\
\hline Dizziness (n; \%) & $0 / 0$ & $\mathrm{I} / 0(1.3)$ & $0 / 2(2.5)$ & $0 / 1(1.2)$ \\
\hline Cutaneous rash (n; \%) & $0 / 0$ & $0 / 0$ & $0 / 0$ & $0 / 0$ \\
\hline Hypoglycemia (n; \%) & $0 / 0$ & $0 / 0$ & I/0 (1.3) & $0 / 1(1.2)$ \\
\hline Hypotension (n; \%) & $0 / 0$ & $0 / 0$ & $0 / 1(1.3)$ & $1 / 0(1.2)$ \\
\hline \multicolumn{5}{|l|}{ IGT patients (III) } \\
\hline Nausea (n; \%) & $0 / 1(1.2)$ & $0 / 0$ & $0 / 0$ & $0 / 0$ \\
\hline Vomiting (n; \%) & $0 / 0$ & $\mathrm{I} / 0(\mathrm{I} .3)$ & $0 / 0$ & $0 / 0$ \\
\hline Dizziness (n; \%) & $0 / 0$ & $0 / 0$ & $\mathrm{I} / 0(\mathrm{I} .3)$ & $0 / 0$ \\
\hline Cutaneous rash (n; \%) & $\mathrm{I} / 0(\mathrm{I} .2)$ & $\mathrm{I} / 0(\mathrm{I} .3)$ & $0 / 0$ & $0 / 1(1.2)$ \\
\hline Hypoglycemia (n; \%) & $0 / 0$ & $0 / 0$ & $0 / 1(1.3)$ & $1 / 0(1.2)$ \\
\hline Hypotension (n; \%) & $0 / 0$ & $0 / 0$ & $0 / 0$ & $0 / 1(1.2)$ \\
\hline
\end{tabular}

Abbreviations: M, males; F, females; IFG, impaired fasting glycemia; IGT, impaired glucose tolerance; EU, euglycemia.

\section{Conclusions}

The chronic use (4 years) of a food supplement containing alpha lipoic acid is well tolerated, without significant differences between lower and higher dosages and improves glycemic status and lipid profile, but only if administered at high dosage.

\section{Author Contributions}

All authors contributed to data analysis, drafting or revising the article, have agreed on the journal to which the article will be submitted, gave final approval of the version to be published, and agree to be accountable for all aspects of the work.

\section{Disclosure}

The authors report no conflicts of interest for this work and have no relevant affiliations or financial involvement with any organization or entity with a financial interest in or financial conflict with the subject matter or materials discussed in the manuscript. This includes employment, consultancies, honoraria, stock ownership or options, expert testimony, grants or patents received or pending, or royalties. No writing assistance was utilized in the production of this manuscript.

\section{References}

1. Bianchi C, Miccoli R, Penno G, Del Prato S. Primary prevention of cardiovascular disease in people with dysglycemia. Diabetes Care. 2008;31(Suppl 2):S208-S214. doi:10.2337/dc08-s256

2. Coutinho M, Gerstein HC, Wang Y, Yusuf S. The relationship between glucose and incident cardiovascular events. a metaregression analysis of published data from 20 studies of 95,783 individuals followed for 12.4 years. Diabetes Care. 1999;22:233-240. doi:10.2337/ diacare.22.2.233

3. De Fronzo R, Ferrannini E. Insulin resistance: a multifaceted syndrome responsible for NIDDM, obesity, hypertension, dyslipidemia, and atherosclerotic cardiovascular disease. Diabetes Care. 1991;14:173-194. doi:10.2337/diacare.14.3.173

4. Busby RW, Schelvis JPM, Yu DS, Babcock GT, Marletta MA. Lipoic acid biosynthesis: lipA is an iron-sulphur protein. $J$ Am Chem Soc. 1999;121:4706-4707. doi:10.1021/ja990134g 
5. Derosa G, D'Angelo A, Romano D, Maffioli P. A clinical trial about a food supplement containing $\alpha$-lipoic acid on oxidative stress markers in type 2 diabetic patients. Int J Mol Sci. 2016;17(11):1802. doi:10.3390/ijms 17111802

6. Eason RC, Archer HE, Akhtar S, Bailey CJ. Lipoic acid increases glucose uptake by skeletal muscles of obese-diabetic ob/ob mice. Diabetes Obes Metab. 2002;4(1):29-35. doi:10.1046/j.14631326.2002.00171.x

7. European Diabetes Policy Group. A desktop guide to type 2 diabetes mellitus. Diabet Med. 1999;16:716-730. doi:10.1046/j.14645491.1999.00166.x

8. Klose S, Borner K. Enzymatische Bestimmung des Gesamtcholesterins mit dem [Enzymatic dosage of total cholesterolemia by Greiner Selective Analyzer (GSA II)]. J Clin Chem Clin Biochem. 1978;15:121-130.

9. Wahlefeld AW. Triglycerides determination after enzymatic hydrolysis. In: Bergmeyer HU, editor. Methods of Enzymatic Analysis. 2nd English ed. New York: Academic Press; 1974:18-31.

10. Havel RJ, Eder HA, Bragdon JH. The distribution and chemical composition of ultracentrifugally separated lipoproteins in human serum. J Clin Invest. 1955;34:1345-1353. doi:10.1172/JCI103182
11. Friedewald WT, Levy RI, Fredrickson DS. Estimation of the concentration of low density lipoprotein in plasma, without use of the preparative ultracentrifuge. Clin Chem. 1972;18:499-502. doi:10.1093/clinchem/18.6.499

12. Rifai N, Tracy RP, Ridker PM. Clinical efficacy of an automated high-sensitivity C-reactive protein assay. Clin Chem. 1999;45 (12):2136-2141. doi:10.1093/clinchem/45.12.2136

13. Winer BJ. Statistical Principles in Experimental Design. 2nd ed. New York: McGraw-Hill; 1971.

14. Akbari M, Ostadmohammadi V, Lankarani KB, et al. The effects of alpha-lipoic acid supplementation on glucose control and lipid profiles among patients with metabolic diseases: A systematic review and meta-analysis of randomized controlled trials. Metabolism. 2018;87:56-69. doi:10.1016/j.metabol.2018.07.002

15. Okanović A, Prnjavorac B, Jusufović E, Sejdinović R. Alpha-lipoic acid reduces body weight and regulates triglycerides in obese patients with diabetes mellitus. Med Glas (Zenica). 2015;12(2):122-127.

16. Parente E, Colannino G, Picconi O, Monastra G. Safety of oral alpha-lipoic acid treatment in pregnant women: a retrospective observational study. Eur Rev Med Pharmacol Sci. 2017;21(18):4219-4227.

\section{Publish your work in this journal}

Drug Design, Development and Therapy is an international, peerreviewed open-access journal that spans the spectrum of drug design and development through to clinical applications. Clinical outcomes, patient safety, and programs for the development and effective, safe, and sustained use of medicines are a feature of the journal, which has also been accepted for indexing on PubMed Central. The manuscript management system is completely online and includes a very quick and fair peer-review system, which is all easy to use. Visit http://www. dovepress.com/testimonials.php to read real quotes from published authors. 